\title{
Polarization Properties of Active Galactic Nuclei
}

\author{
J. H. Hough \\ Department of Physical Sciences, University of Hertfordshire, Hatfield, \\ $A L 109 A B, U K$
}

\begin{abstract}
.
One of the main uses of polarimetry over the last decade, has been to identify the nature of AGN that are normally hidden from direct view by an optically and geometrically thick torus, and thereby to unify different classes of AGN. Of growing importance is the role that polarimetry can play in our understanding of the properties and structure of AGN on a size scale which cannot be resolved directly. We review the progress being made in understanding the role of disks, extended scattering regions, and the obscuring torus in $\mathrm{AGN}$.
\end{abstract}

\section{Introduction}

Polarimetry has played a key role in developing our understanding of active galactic nuclei (AGN). In some cases it establishes the basic radiation mechanism; it can provide a view of normally obscured regions, and a comparison of total and (scattered) polarized flux provides views of a source from different angles; it is possible to study the geometrical and velocity relationship between source, scatterer and observer, without spatially resolving the source; and the presence and direction of magnetic fields can also be determined. Examples of all these diagnostics will be provided in this review, which includes a brief outline of sources where synchrotron emission is important, and then covers the role played by polarimetry in the unification of type I and type II AGN, in the imaging of the torus in NGC1068, in attempting to establish the beaming pattern of the continuum radiation in radio-quiet AGN, and in discriminating between different accretion disk models. In the space provided it is impossible to cover the complete role of polarimetry and many of the examples used are drawn from our own work. For additional information the reader should refer to the various cited papers, and to useful reviews by Antonucci (1993) and Miller (1993).

\section{Core-dominated radio sources}

The observed emission from flat-spectrum, core-dominated, radio sources is dominated by doppler-boosted synchrotron emission from relativistic jets, producing high and variable degrees of polarization (typically 5 to $50 \%$ ). This masks any contributions from other AGN components such as thermal emission from an accretion disk and hot dust, as well as starlight from a host galaxy, producing 
an essentially featureless continuum. Such objects have traditionally been referred to as BL Lacertaes. Core-dominated radio sources, still with high and variable polarization but with clear evidence of other emission features, such as the Big Blue Bump covering the UV and optical wavelengths (presumed to be thermal emission from an accretion disk with temperatures $\sim 30,000-50,000 \mathrm{~K}$ ), the Red Bump at near-infrared wavelengths (presumed to be from hot dust at temperatures upto $\sim 2000 \mathrm{~K}$ ), and with broad emission lines, are referred to as Highly Polarized Quasars (HPQs), or Optically Violently Variables (OVVs). All of these additional features have degrees of polarization substantially less than the continuum synchrotron radiation, and may well be unpolarized. An extensive and recent review of Unified Schemes for Radio-Loud Active Galactic Nuclei is given in Urry \& Padovani (1995).

\section{Unification of type I and type II galaxies}

Over the last decade polarimetry of AGN has focussed much more on spectropolarimetry and this has achieved notable success, particularly in the study of low luminosity type II radio-quiet AGN (type II Seyferts). First established by Antonucci \& Miller (1985) through observations of NGC1068 (see also Bailey et al 1988, Young et al 1995), the current unified model of Seyfert galaxies has all such galaxies hosting a type I nucleus, i.e. a featureless continuum source and a broad-line region (BLR), surrounded by a geometrically thick torus which obscures our view of the nuclear regions for the type II Seyferts, but not the type Is. For the type IIs radiation escaping along the polar axis of the torus can be scattered towards us, revealing a type I spectrum in the polarized flux spectrum. A nice example of this is shown in Figure 1 for IRAS11058-1131 (Young et al 1993 and 1996a). This phenomenon has been observed in about 20 type II Seyfert galaxies (Miller \& Goodrich 1990, Tran, Miller \& Kay 1992, Young et al 1993 , Hines \& Wills 1993, Kay 1994, Young et al 1996a\&b), and in 3 narrow-line radio galaxies (Antonucci 1984, Inglis et al 1993, Young et al 1996c\&d).

\section{The obscuring torus and magnetic fields}

In the UV and optical, the polarized flux is dominated by photons from the AGN continuum, scattered by electrons in the cone-like structures observed in high-ionization emission line images (Pogge 1988, Unger et al 1992). These cones are generally aligned with the large-scale radio axis (Wilson \& Ulvestad $1983,1987)$, and are presumably formed by ionizing photons from the nuclear continuum, radiation bounded by the nuclear torus, although some intrinsic beaming of the nuclear continuum source may be possible (see section 5). In polarized light the scattering produces a classical reflection nebula (Scarrott et al 1991), which at optical wavelengths is one-sided, as the dusty disk of the host galaxy obscures radiation from the far cone at these wavelengths. In the nearinfrared, however, the true bipolar nature of the reflection nebulosity is revealed (see Figure 2, and Young et al 1996e, Packham et al 1996). In the J $(1.2 \mu \mathrm{m})$ and H-bands $(1.6 \mu \mathrm{m})$, the torus itself, at least for NGC1068, can be detected in polarized flux, with scattered radiation from the far-cone back-illuminating the obscuring torus, which is then seen in silhouette as the torus absorbs this 


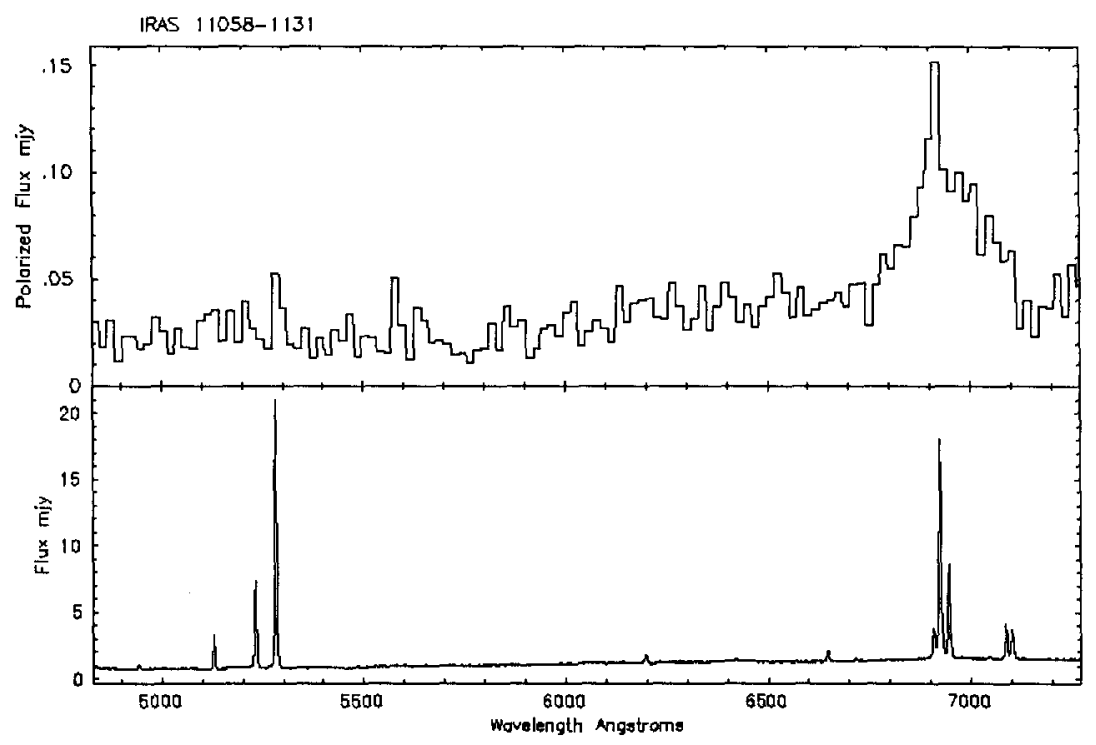

Figure 1. Spectropolarimetric data for IRAS11058-1131, showing the total flux spectrum (lower) and polarized flux spectrum (upper). From Young et al., 1993

scattered radiation (see Figure 3, and Young et al 1996e). From a geometrical analysis, it is possible to calculate the inclination and position angle of the torus ( $42 \pm 4^{\circ}$, and $32 \pm 3^{\circ}$ respectively), entirely consistent with the values derived from models of the scattered radiation in the ionization cones (Young et al 1995). It is also possible to calculate the diameter of the torus, $\sim 200 \mathrm{pc}$, much larger than proposed previously for the tori in AGN (for example, Pier \& Krolik 1993). This diameter is, however, entirely consistent with the value derived from recent modelling of the infrared SED of the torus (Efstathiou, Hough \& Young 1995), the size of the region from which HCN emission is observed (Tacconi et al 1994), and similar to the size required to explain the differences between type I, type II and intermediate type Seyferts (Maiolino et al 1995, Maiolino \& Rieke 1995).

In the K-band $(2.2 \mu \mathrm{m})$, the nuclear polarized flux spectrum of NGC1068, and several other type II Seyferts with hidden type I nuclei, cannot be explained by the scattering of a type I spectrum alone (Young et al 1995, 1996a). In addition a contribution from dichroic absorption of the radiation from the IRemitting regions of the AGN, through the torus is required. For NGC1068, the extinction to this region is $A_{v} \sim 45 \mathrm{mag}$, substantially less than the extinction to the BLR and the nuclear continuum source. There are two requirements. First the majority of the K-band flux arises from a region which is separate from the UV and optical continuum source. While the most obvious source of this radiation might be the inner wall of the torus, illuminated directly by the nuclear continuum, Efstathiou, Hough \& Young (1995), in their radiative transfer model 


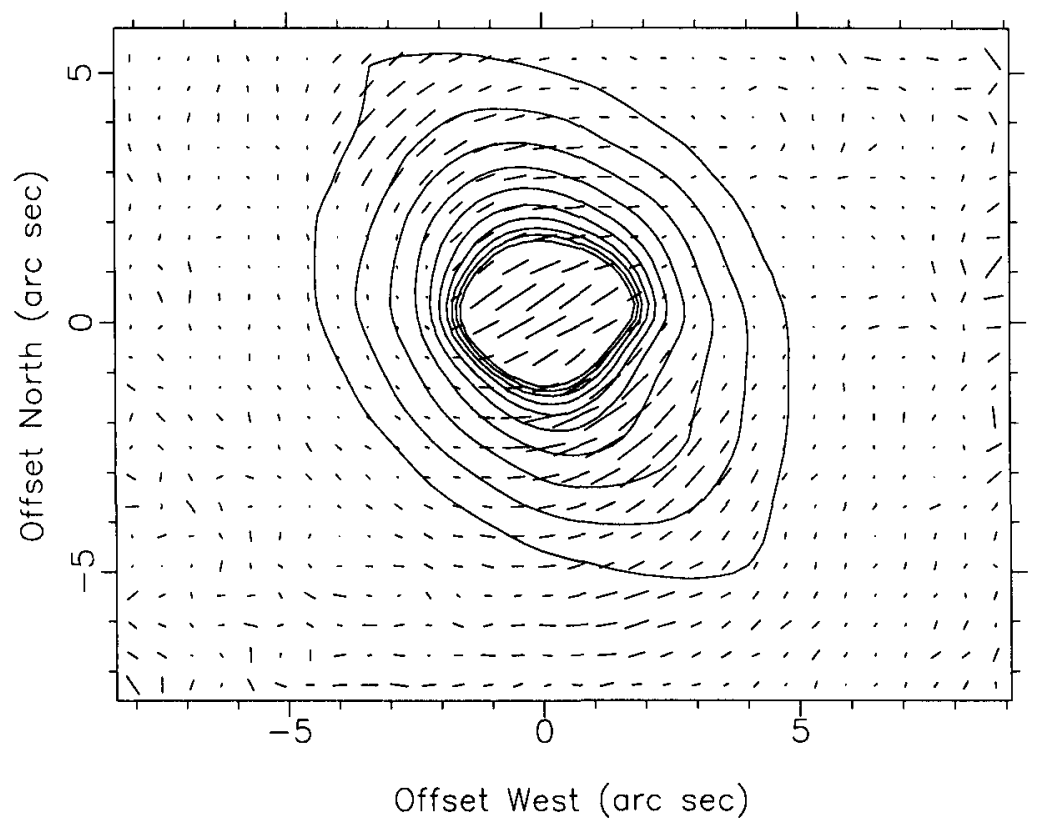

Figure 2. Polarization vectors in the $\mathrm{H}$ band, for NGC1068, superimposed upon the total intensity contours; the length of a $100 \%$ polarization vector would be 5.72 arcsec. The bipolar nature of the reflection nebulosity can be easily observed at near-IR wavelengths. From Young et al., 1996e.

which uses a tapered disk geometry for the torus, suggest that hot dust in the funnel of the torus is more important.

Second, in order for the radiation to be polarized, the dust grains within the torus must be aligned (for details of grain alignment mechanisms see Lazarian $1996 \mathrm{a}, \mathrm{b}, \mathrm{c}$ and references therein). In the presence of a magnetic field, the grains will align with their short axis along the direction of the field, and produce polarization (by absorption) with the E-vector parallel to the magnetic field. For those objects studied by Young et al $(1995,1996 \mathrm{a})$, the direction of $\mathrm{B}$ is perpendicular to the radio axis, to within $\sim 20^{\circ}$, and thus adds to the sense of the polarization produced by scattering in the cones. This means that, as projected onto the sky, the direction of $B$ is in the plane of the torus, at least at a height from the torus equator of $\sim 1-2 \mathrm{pc}$, where we believe the near-infrared emission is viewed. Thus if the magnetic field is overall poloidal, it must be twisted around sufficiently, at this height, to appear close to the torus plane.

In general terms this is similar to the results of calculations by Kartje (1995), who uses the Konigl \& Kartje (1994) model in which centrifugally driven winds from the surfaces of a magnetized accretion disk are responsible for removing the angular momentum of the accreted matter and for producing the bipolar outflows and jets. In their picture the torus is identified with the outer regions of the winds where dust uplifted from the disk surfaces by gas-grain collisions is embedded in the outflow. Kartje proposes that some degree of grain alignment 


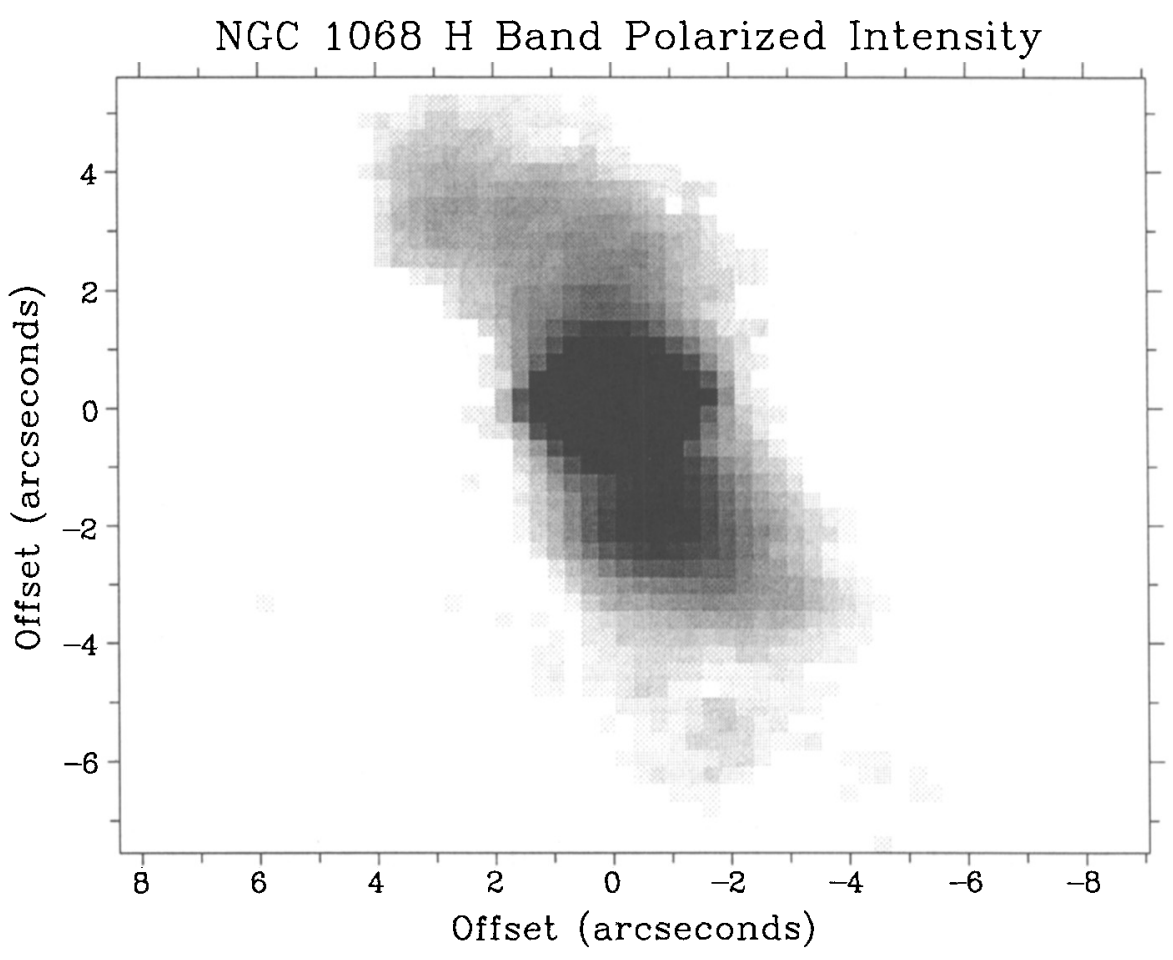

Figure 3. A grey-scale polarized flux image of NGC1068 in the $\mathrm{H}$ band. In this negative image, the obscuring torus is seen as a light band, between the forward pointing scattering cone to the north and the back pointing scattering cone to the south. From Young et al., $1996 \mathrm{e}$.

would occur in the dense torus, and this would be most efficient near the surfaces of a torus cloud or dusty wind directly exposed to the central continuum. He suggests that because the magnetic field lines are primarily toroidal throughout much of the wind, dichroism from magnetically aligned grains tends to reinforce the scattering induced polarization, at least for relatively large viewing angles. As the wind model produces a marked density stratification in the vertical direction Kartje predicts a significant dichroic contribution at optical wavelengths, which is not supported by the modelling of Young et al $(1995,1996 \mathrm{a})$.

\section{Beaming pattern of continuum radiation from radio-quiet AGN}

While radio-loud AGN contain relativistically boosted jets (only resolved at optical wavelengths for a few objects such as M87 and 3C273), the intrinsic beaming pattern of radio-quiet objects, as opposed to shadowing by the torus, is unknown. 
Miller, Goodrich \& Mathews (1991), for NGC1068, suggested that for reasonable torus parameters, the amount of energy intercepted by the torus was less than that going into the ionization cones, for an intrinsically isotropic continuum source. The model of Esfstathiou, Hough \& Young (1995), which requires a component of optically thin dust in the torus funnel, in order to account for the observed SED (see section 4), also requires that the heating source for the dust is anisotropic, with power along the poles of the torus about 6 times that along the equatorial direction. Assuming the heating radiation arises from an accretion disk, this would argue in favour of a geometrically thick disk, where emission is dominated by flux originating deep inside the funnel, with the harder radiation then emitted preferentially along the funnel axis.

Young et al (this conference), have used spectropolarimetry to compare the equivalent widths of scattered broad-lines across the ionization cone. If the broad-line and continuum photons see the same scatterers, then the change of equivalent width across the cone will map any change in the beaming pattern of the continuum and line radiation. Although only a few points have been sampled, the equivalent width is less on the cone-axis than it is along the coneedge, as expected for a continuum source which is beamed along the cone-axis.

\section{The accretion disk}

\subsection{Thin accretion disks}

Thermal radiation from geometrically thin accretion disks with a temperature of $\sim 30,000 \mathrm{~K}$ has been a popular explanation for the Big Blue Bump, observed in many AGN. In practice the bump is broader than a single blackbody curve, indicating contributions from a disk surface with a range of temperatures. The region of the disk in which UV radiation arises will be dominated by electron scattering opacity, which should then produce linear polarization.

For an optically thick, scattering planar atmosphere, degrees of polarization range from zero for a face-on view to $\sim 12 \%$ for an observer at grazing angle. The position angle of polarization (PA) is perpendicular to the disk rotational axis, projected onto the sky plane, as defined from the radio axis (e.g. Webb \& Malkan 1986, Coleman \& Shields 1990). Unfortunately, radio-quiet type I systems (i.e. where the accretion disk is presumably viewed directly), and quiescent radio quasars, show small polarizations (typically below $2 \%$ ), and have a PA which is parallel to the disk axis (Brindle et al 1990). The low degrees of polarization in themselves are not necessarily a strong argument against the thin accretion disk model, since the accretion disk is likely to be observed directly at relatively small inclinations (at large inclinations it would be obscured by the nuclear torus), but the observed PAs are.

Coleman \& Shields (1990) have attempted to overcome the problem by proposing a thin disk with a surface roughened by local irregularities, such as surface waves, so that a substantial fraction of the emitted light scatters off neighbouring protrusions before leaving the disk. In this case the polarization can be weak and parallel to the disk axis.

Laor, Netzer \& Piran (1990) have considered thin accretion disk models which are sufficiently dense that they have significant absorption opacity as well as electron scattering opacity. They argue that this will produce a significant 
wavelength dependence in the degrees of polarization. Specifically, their model predicts a significant polarization in the $1000-2000 \AA$ range, where the disk radiation should be significantly polarized, but is then substantially reduced below the Lyman edge, where absorption opacity dominates. Again, the PA of polarization should be perpendicular to the radio axis, contrary to observations, a point which this model does not address. Koratkar et al (1995, and this conference) and Antonucci et al (1996), have presented spectropolarimetry data for a number of quasars covering the wavelength region adjacent to the Lyman edge. In general the polarizations observed are low, and in a number of cases with no significant wavelength dependence. A few objects, however, showed a very large increase in polarization, with decreasing wavelength, shortward of the Lyman edge, reaching values as high as $20 \%$ (for PG $1630+377$, Koratkar et al 1995). These results are quite contrary to the predictions of the Laor, Netzer and Piran (1990) model, although Blaes (this conference), and Blaes \& Agol (1996) argue that it is possible to explain the observed polarization behaviour with more realistic thin disk models, including - for example - the effects of Faraday rotation. Nonetheless, it is not clear that the problem with the position angle of polarization can be readily resolved for the standard thin disk model.

Although it is possible to modify the standard thin accretion disk model to obtain low degrees of polarization and the correct PA (e.g. the corrugated surface model of Coleman \& Shields (1990), and a suitably constructed extended source function would no doubt suffice), it would seem appropriate to consider different types of accretion disks, e.g. a geometrically thick accretion disk, which will also provide intrinsic anisotropy in the beaming pattern of the continuum radiation (see the previous section).

\subsection{Thick accretion disks (tori)}

Coleman \& Shields (1990, see also Kartje 1995), considered a thick accretion disk, where emission is dominated by flux originating deep inside the funnel, and the radiation scatters many times from the sides of the funnel before escaping to the observer. For a narrow funnel (half-angle $9^{\circ}$ ), the degrees of polarization in the centre of the beam are a few tenths of a percent and parallel to the disk axis, and the polarization does not exceed $0.5 \%$ within the beam containing $90 \%$ of the luminosity. For a wide funnel (half-angle $23^{\circ}$ ), the behaviour of the polarization is more complex, but near the disk axis is very small although this time it is perpendicular to the disk axis (see Figure 7 of Coleman \& Shields 1990).

\section{Does broad emission line gas reside in the disk?}

A few Broad-Line Radio Galaxies (BLRGs) are observed to have double-peaked Balmer emission lines, with the blue shifted peak generally larger. The relativistic disk model of Chen \& Halpern (1989) has generally been successful in reproducing the observed line profiles. In order to overcome the problem of a thin disk subtending a sufficient solid angle at the central source for the required degree of photoionization, they assume the inner part of the disk is a geometrically thick hot-ion torus which then illuminates the outer parts of the disk with ionizing radiation to produce the observed broad-lines. The asymmetry 
between the red and blue shifted lines arises from the doppler boosting of the line-emission from the gas moving towards the observer.

An important part of the Chen \& Halpern model is electron scattering in the disk atmosphere which can produce the observed line broadening. It is then possible to predict the broad-line polarization expected to arise from the electron scattering. They predict a polarization which is orthogonal to the disk axis and with the polarized flux in the red peak equal to or slightly larger than in the blue peak (Chen \& Halpern 1990).

Antonucci, Hurt \& Agol (1996), Corbett et al (1996, and this conference), have shown that the line profiles seen in polarized light do not agree with the model predictions. The polarized line profiles are single-peaked with the PA parallel to the disk axis. It might still be possible to rescue the disk model by assuming that our direct view of the broad-lines is different to that of the scatterers. For example, for Arp102B Chen \& Halpern (1989) find a best fit to the total flux line profiles occurs for a disk viewed at an inclination of about $30^{\circ}$, however, if the scatterers are located on the disk axis, then they do not see a double-peaked profile (Corbett et al 1996, and this conference). To get the $\mathrm{PA}$ of polarization correct requires the scattering to be taking place close to the source of the broad-lines. One possibility is that the scattering is taking place off electrons in the base of the relativistic jet.

A strong argument against the disk model comes from the observation that in total flux 3C390.3 has occasionally had a brighter red than blue peak, which cannot be reproduced by the relativistic disk model. Also, analysis of the recently appeared double peaked line profile in PicA (Halpern \& Eracleous 1994), shows that when the previous epoch $\mathrm{H} \alpha$ profiles are subtracted, two completely separated peaks are left, and the absence of any low velocity components is not easy to explain with the disk model.

\section{Conclusions}

Polarimetry has played a key role in our understanding of AGN, and in particular in the development of unified theories. Most progress has been made in unifying type I and type II radio quiet AGN (the Seyfert galaxies), but even for these there are a number of unanswered questions. For example, not all type II AGN show polarized broad lines in the optical polarized flux spectrum. Does this indicate that type II nuclei really do exist, or is it difficult to detect the scattered broad lines in these cases? Such problems might arise if there is a dearth of scatterers, or the scatterers are themselves obscured from direct view. Nearinfared spectroscopy can be used to detect broad lines which might be viewed directly through the torus, and near-infrared spectropolarimetry can be used to detect scattered broad lines which might be obscured at optical wavelengths. Even when detected in the total flux spectrum, spectropolarimetry is required to determine whether the near-infrared broad lines are indeed observed directly through the torus or are entirely seen in scattered light. This distinction is clearly important in establishing the optical thickness of the torus.

Less progress has been made in studying the radio-loud type I and type II AGN, although with the new generation of larger telescopes spectropolarimetry 
at both optical and near-infrared wavelengths will be available for a much larger number of objects, and thus unification theories can be better tested.

While most observations have been of type II objects, polarimetry can play a very important role in studying type I objects where the central regions can be viewed directly. When some of the radiation is scattered to us, for example by the electron atmospheres of accretion disks, off electrons in radio jets, off dust grains close to the BLR, then polarimetry can provide information on the geometry and nature of the nuclear regions. In particular polarimetry is a valuable aid in testing specific models of accretion disks and the geometry of the BLR.

\section{References}

Antonucci R. R. J., Unified Models for Active Galactic Nuclei and Quasars, Annual Reviews of Astronomy \& Astrophysics, 1993

Antonucci R. R. J., 1984, ApJ, 278, 499

Antonucci R. R. J. \& Miller J., 1985, ApJ, 297, 621

Antonucci R. R. J., Geller R., Goodrich R .W. \& Miller J. S., 1996, ApJ, in press

Antonucci R., Hurt T. \& Agol E., 1996, ApJ, 456, L25

Bailey J., Axon D. J., Hough J. H., Ward M. J., McLean I. S., \& Heathcote S., 1988, MNRAS, 234, 899

Blaes O. \& Agol E., 1996, ApJ, 469, L41

Brindle C., Hough J. H., Bailey J. A., Axon D. J., Ward M. J., Sparks W. B. \& McLean I. S., 1990, MNRAS, 244, 577

Chen K. \& Halpern J. P., 1989, ApJ, 344, 115

Chen K. \& Halpern J. P., 1990, ApJ, 354, L1

Coleman H. H. \& Shields G. A., 1990, ApJ, 363, 415

Corbett E. A., Robinson A., Young S., Hough J. H. \& Axon D. J., 1996, MNRAS, submitted

Efstathiou A., Hough J. H. \& Young S., 1995, MNRAS, 277, 1134

Halpern J. P. \& Eracleous M., 1994, ApJ, 433, L17

Hines D. C. \& Wills B. J., 1993, ApJ, 415, 82

Inglis M. D., Brindle C., Hough J. H., Young S., Axon D. J., Bailey J. A. \& Ward M. J., 1993, MNRAS, 263, 895

Kartje J. F., 1995, ApJ, 452, 565

Kay L. E., 1994, ApJ, 430, 296

Konigl A. \& Kartje J. F., 1994, ApJ, 434, 446

Koratkar A., Antonucci R. R. J., Goodrich R. W., Bushouse H. \& Kinney A. L., 1995, ApJ, 450, 501

Laor A., Netzer H. \& Piran T., 1990, MNRAS, 242, 560

Lazarian A., 1996a, b \& c: Mechanical Alignment of Suprathermal Grains, page 425; Gold Alignment, page 433; Paramagnetic Alignment, page 438, in Proc. of the conference Polarimetry of the Interstellar Medium, ASP Conference Series, vol 97. 
Maiolino R. \& Rieke G. H., 1995, ApJ, 454, 95

Maiolino R., Ruiz M., Rieke G. H. \& Keller L. D., 1995, ApJ, 446, 561

Miller J. S., The Unification of Active Galaxies: Seyferts \& Beyond, The First Stromlo Symposium: The Physics of Active Galaxies, Astronomical Society of the Pacific Conference Series, 1993, 54, 149

Miller J. S. \& Goodrich R. W., 1990, ApJ, 355, 456

Miller J. S., Goodrich R. W. \& Mathews W. G., 1991, ApJ, 378, 47

Packham C., Young S., Hough J. H., Axon D. J. \& Bailey J. A., 1996, MNRAS, submitted

Pier E. A. \& Krolik J. H., 1993, ApJ, 418, 673

Pogge R., 1988 ApJ, 328, 519

Scarrott S. M., Rolph C. D., Wolstencroft R. W., \& Tadhunter C. N., 1991, MNRAS, 249, L16

Tacconi L. J., Genzel R., Bleitz M., Cameron M., Harris A. I., \& Madden S., 1994, ApJ, 426, L77

Tran H. D., Miller J. S. \& Kay L. E., 1992, ApJ, 397, 452

Unger S. W., Lewis J. R., Pedlar A. \& Axon D. J., 1992, MNRAS, 258, 371

Urry C. M. \& Padovani P., 1995, PASP, 107, 803

Webb W. \& Malkan M., 1986, In: The Physics of Accretion Onto Compact Objects, p15, eds. Mason K O, Watson M G \& White N E, SpringerVerlag, Berlin

Wilson A. S. \& Ulvestad J. S. 1983, ApJ, 275, 8

Wilson A. S. \& Ulvestad J. S. 1987, ApJ, 319, 105

Young S., Hough J. H., Bailey J. A., Axon D. J. \& Ward M. J., 1993, MNRAS, 260, L1

Young S., Hough J. H., Axon D. J., Bailey J. A. \& Ward M. J., 1995, MNRAS, 272,513

Young S., Hough J. H., Efstathiou A., Wills B. J., Bailey J. A., Ward M. J. \& Axon D. J., 1996a, MNRAS, 281, 1206

Young S., Hough J H., Axon D J., Ward M. J. \& Bailey J. A., 1996b, MNRAS, 280,291

Young S., Hough J. H., Efstathiou A., Wills B. J., Axon D. J., Bailey J. A. \& Ward M. J., 1996c, MNRAS, 279, L72

Young S., Axon D. J., Hough J.H., Fabian A.C. \& Ward M. J., 1996d, MNRAS, submitted

Young S., Packham C., Hough J. H. \& Efstathiou A., 1996e, MNRAS, in press 